УДК 577.13

\title{
ФИТОЭКДИСТЕРОИДЫ КОРНЕЙ SILENE FRIVALDSZKYANA HAMPE
}

(ㄱ Л.Н. Зибарева ${ }^{*}$, О.В. Волкова ${ }^{1}$, С.В. Морозов $^{2}$ Е.И. Черняк ${ }^{2}$

${ }^{1}$ Томский государственный университет, Сибирский ботанический сад, пр. Ленина, 36, Томск, 634050 (Россия), e-mail: zibareva.lara@yandex.ru ${ }^{2}$ Новосибирский институт органической химии СО РАН, пр. Лаврентьева, 9 , Новосибирск, 630090 (Россия)

Из корней интродуцированных в Западную Сибирь Silene frivaldszkyana (Caryophyllaceae) выделены экдистероиды: 20-гидроксиэкдизон, полиподин В, интегристерон А, 2-дезокси-20-гидроксиэкдизон, 2-дезоксиэкдизон, 20,26-дигидроксиэкдизон, 26-гидроксиполиподин В, 20-гидроксиэкдизон-25- $\beta$-D-глюкозид. Структуры идентифицированы с помощью методов ЯМР-, масс-спектрометрии, ВЭЖХ. Наличие 26-гидроксиинтегристерона А в корнях установлено при сравнительном ВЭЖХ-анализе с выделенным соединением из надземной части Silene frivaldszkyana. Показано, что исследуемый вид Silene синтезирует характерные для данного рода экдистероиды: 2-дезокси-20гидроксиэкдион, 2-дезоксиэкдизон, 20-гидроксиэкдизон, полиподин В, интегристерон А и является богатым источником их 26-гидрокси производных. Наряду с распространенными экдистероидами в видах рода Silene впервые в исследуемом виде выделен 20-гидроксиэкдизон-25- $\beta$-D-гликозид.

Сравнительный анализ состава экдистероидов в надземной и подземной частях показал, что корни содержат более богатый их состав, видимо, это связано с тем, что корни многолетних растений аккумулируют эти соединения. А 20-гидроксиэкдизон-25- $\beta$-D-глюкозид, вероятно, является запасным веществом мажорного компонента - 20гидроксиэкдизона.

Ключевые слова: фитоэкдистероиды, идентификация, корни Silene frivaldszkyana.

Исследование выполнено при финансовой поддержке РГНФ/РФФИ и администрации Томской области в рамках научного проекта № 16-44-700634.

\section{Введение}

Растения синтезируют множество стероидных соединений, присущих не только растительному, но и животному миру. Наиболее широко распространены в мировой флоре экдистероиды, которые являются аналогами гормонов линьки и метаморфоза насекомых. Экдистероиды по химической природе принадлежат к полиоксистероидам с гидроксильными группами в стероидном ядре и боковой цепи, циссочленением между циклами А и В, содержащими 7-ен-6-он хромоформную группу, 14-ОН. В настоящее время известно о широком распространении этих стероидов в растениях семейства Caryophyllaceae (роды Lychnis, Silene, Petrocoptis и др.).

Silene frivaldszkyana Натре. - один из перспективных видов по содержанию и составу фитоэкдистероидов, впервые рекомендованный в качестве источника этих БАВ [1, 2]. В процессе многолетнего изучения в Сибирском ботаническом саду Томского государственного университета (СБС ТГУ) вида Silene

Зибарева Лариса Николаевна - доктор химических наук, заведующая лабораторией фитохимии,

e-mail: zibareva.lara@yandex.ru

Волкова Ольга Владимировна - старший лаборант, e-mail: olgavolkova2510@ rambler.ru

Морозов Сергей Владимирович - кандидат химических наук, заведующий лабораторией,

e-mail:morozov@nioch.nsc.ru

Черняк Елена Ильинична - старший научный сотрудник, e-mail: chernyak@nioch.nsc.ru frivaldszkyana, являющегося эндемиком Балканского полуострова, показано, что растения способны успешно адаптироваться в Западной Сибири и произрастать на протяжении 17 лет. Растения проходят полный цикл развития, получен ряд поколений, сохраняющих способность синтезировать фитоэкдистероиды.

Установлено, что в надземной части Silene frivaldszkyana синтезируются 20-гидроксиэкдизон,

\footnotetext{
* Автор, с которым следует вести переписку.
} 
полиподин В, 2-дезокси-20-гидроксиэкдизон, 2-дезоксиэкдизон, интегристерон А, 26-гидроксиполиподин В, 20,26-гидроксиэкдизон, 26-гидроксиинтегристерон А $[3,4]$. Последнее соединение является новым и впервые выделенным. Структуры выделенных соединений установлены с помощью ЯМР-, массспектрометрии, ВЭЖХ. Информация о выделенных экдистероидах и источнике включена в международную базу данных Ecdybase.org [5]. Отличительной особенностью состава этого вида является синтез ряда 26 -оксипроизводных экдистероидов $[3,6]$. Поскольку корни этого ценного вида отличаются высоким содержанием экдистероидов, важно было изучить их состав.

Цель настоящего исследования - изучение состава экдистероидов корней Silene frivaldszkyana, интродуцированного в Сибирском ботаническом саду ТГУ.

\section{Экспериментальная часть}

Выделение экдистероидов проводили из подземной части растений, интродуцированных в СБС ТГУ с 1994 г. Растительное сырье является вторым поколением от маточных растений, семена для которых получены из ботанического сада Галле (Германия).

Корни Silene frivaldszkyana (250 г) экстрагировали пятикратно 70\% этиловым спиртом. Экстрагент отгоняли под вакуумом при температуре $50{ }^{\circ} \mathrm{C}$ с помощью вакуумного ротационного испарителя IKA HB 10 digital (Германия). Концентрированный экстракт разбавляли водой в соотношении $1: 2$, отфильтровывали. Фильтрат очищали от липофильных веществ н-гексаном. Сумму экдистероидов из очищенного фильтрата извлекали н-бутанолом. Бутанольную фракцию сконцентрировали до смолообразного состояния.

Затем полученную бутанольную смесь фракционировали системами растворителей хлороформ этиловый спирт в соотношениях $7: 1 ; 4: 1 ; 2: 1$. Полученные фракции сконцентрировали на ротационном испарителе при температуре $50{ }^{\circ} \mathrm{C}$. Концентрированные фракции, обогащенные неполярными, средне полярными и полярными экдистероидами, подвергали многократному разделению на колонках с силикагелем марки КСК (Россия) с размером частиц 0,10-0,16 мм. В процессе хроматограграфирования в качестве элюентов использовали системы растворителей хлороформ - этанол: 1) $9: 1$;) $7: 1$; 3) $5: 1$; 4) $4: 1$; 5) $2: 1 ; 6) 1: 1$. Контроль над разделением веществ осуществляли с помощью TCX на пластинках TLC Silica gel $60 \mathrm{~F}_{254}$ Merk. Идентификацию выделенных экдистероидов Silene frivaldszkyana проводили с помощью методов ВЭЖХ/УФ, ВЭЖХ/МС и ЯМР-спектроскопии.

ВЭЖХ/УФ-анализ выполнен на жидкостном хроматографе Agilent 1100 с диодноматричным детектором. Хроматографическая колонка ZORBAX Eclipse XDB C8; 4,6×150 мм, размер зерна сорбента 5 мкм. Градиентное элюирование вели системой растворителей метанол - 0,1\% трифторуксусная кислота от 0 до $100 \%$ метанола; скорость элюирования 0,8 мл/мин. Аналитическая длина волны $\lambda_{\max }=254$ нм для регистрации фитоэкдистероидов.

ВЭЖХ/MC-анализ проводили на жидкостном хроматографе Agilent 1200 с диодно-матричным детектором и гибридным квадруполь-время пролетным масс-спектрометром micrOTOF-Q (фирма Bruker). Колонка: Zorbax XDB-C8, 2,1×50 mm. Элюент: $\mathrm{H}_{2} \mathrm{O}-\mathrm{ACN}$ (линейный градиент содержания ACN от 5 до $40 \%$ с 0 до 20 мин). Скорость потока: 0,4 мл/мин. Рабочие параметры масс-детектирования: методы ионизации: химическая ионизация и электростатическое распыление при атмосферном давлении (APCI pos. и API-ES); сканирование положительных и отрицательных ионов в диапазоне $\mathrm{m} / \mathrm{z}=200-800$; поток газаосушителя (азот): 4 л/мин, температура $240{ }^{\circ} \mathrm{C}$, температура на распылителе $-390{ }^{\circ} \mathrm{C}$. Спектры ЯМР зарегистрированы на приборах «Bruker AV 400».

\section{Обсуждение результатов}

ВЭЖХ/УФ бутанольной фракции, выделенной из корней S. frivaldszkyana, свидетельствует о более богатом составе экдистероидов, чем в надземной части, идентификация которых была осуществлена в лаборатории профессора Р. Лафона (университет П. и М. Кюри, Париж) [3]. В бутанольной фракции надземной части Silene frivaldszkyana обнаружено 10 экдистероидов, тогда как в бутанольной фракции экстракта корней Silene frivaldszkyana - 14 экдистероидов.

В результате адсорбционной хроматографии на колонках и препаративной ВЭЖХ выделено 8 соединений. Мажорным компонентом экдистероидной суммы исследуемого вида является соединение со временем удерживания 9,727 мин, имеющее характерный для экдистероидов УФ-спектр ( $\lambda=248$ нм) и масс-спектр с характерной фрагментацией $\left(\mathrm{M}^{+} 481\right)$, что соответствует структуре 20-гидроксиэкдизона $(\mathbf{I})$. Это подтвержда- 
ется и спектром ПМР ( $\mathrm{CD}_{3} \mathrm{OD}, \delta$, м.д.): 0,884 (3H, c, $\left.\mathrm{CH}_{3}-18\right), 0,964\left(3 \mathrm{H}, \mathrm{c}, \mathrm{CH}_{3}-19\right), 1,202\left(3 \mathrm{H}, \mathrm{c}, \mathrm{CH}_{3}-21\right)$, 1,190 (3H, с, $\left.\mathrm{CH}_{3}-26,27\right), 1,753$ (2Н, д, Н-1), 5,822 (1Н, д, Н-7), 1,295 (2Н, д, Н-23), 3,843 (1Н, т, Н-2), 3,873 (1H, т, H-3), 1,786 (2H, т, H-4), 2,386 (1H, т, H-5, H-17), 3,149 (1H, т, H-9), 1,425 (2H, т, H-24).

Полиподин В (II), mp. 215-217 ${ }^{\circ} \mathrm{C} .{ }^{1} \mathrm{H}-\mathrm{NMR}\left(\mathrm{CDCl}_{3}+\mathrm{CD}_{3} \mathrm{OD}, \mathrm{ppm}\right): \delta 0,73\left(3 \mathrm{H}, \mathrm{s}, \mathrm{CH}_{3}-18\right), 0,76(3 \mathrm{H}$, s, CH3-19), 1,06 (3H, s, $\left.\mathrm{CH}_{3}-21\right), 1,09\left(6 \mathrm{H}, \mathrm{s}, \mathrm{CH}_{3}-26\right.$ and $\left.\mathrm{CH}_{3}-27\right), 2,19(1 \mathrm{H}, \mathrm{t}$-like, $J=8,4 \mathrm{~Hz}, \mathrm{H}-17), 2,99(1 \mathrm{H}$, t-like, $J=8,1 \mathrm{~Hz}, \mathrm{H}-9), 3,22$ (1H, m, H-22), 3,76 (1H, m, H-2), 3,85 (1H, br s, H-3), 5,79 (1H, s, H-7).

Интегристерон А (III), ${ }^{1} \mathrm{H}-\mathrm{NMR}\left(\mathrm{CDCl}_{3}+\mathrm{CD}_{3} \mathrm{OD}, \mathrm{ppm}\right): \delta 0,81\left(3 \mathrm{H}, \mathrm{s}, \mathrm{CH}_{3}-18\right), 1,05\left(3 \mathrm{H}, \mathrm{s}, \mathrm{CH}_{3}-19\right)$, 1,12 (3H, s, $\left.\mathrm{CH}_{3}-21\right), 1,15\left(6 \mathrm{H}, \mathrm{s}, \mathrm{CH}_{3}-26\right.$ and $\left.\mathrm{CH}_{3}-27\right), 2,26(1 \mathrm{H}, \mathrm{t}-\mathrm{like}, J=7,7 \mathrm{~Hz}, \mathrm{H}-17), 2,55$ (1H, m, H-5), 2,93 (1H, m, H-9), 3,30 (1H, m, H-22), 3,82 (2H, m, H-1 and H-2), 4,02 (1H, br s, H-3), 5,79 (1H, s, H-7). ESMS (-ve): m/z (\% rel. intensity) 495,9 [M-H] $]^{-}(100)$.

2-Дезокси-20-гидроксиэкдизон (IV), ${ }^{1} \mathrm{H}-\mathrm{NMR}\left(\mathrm{CDCl}_{3}+\mathrm{CD}_{3} \mathrm{OD}, \mathrm{ppm}\right): \delta 0,79\left(3 \mathrm{H}, \mathrm{s}, \mathrm{CH}_{3}-18\right), 0,91$ (3H, s, $\left.\mathrm{CH}_{3}-19\right), 1,13\left(3 \mathrm{H}, \mathrm{s}, \mathrm{CH}_{3}-21\right), 1,16\left(6 \mathrm{H}, \mathrm{s}, \mathrm{CH}_{3}-26\right.$ and $\left.\mathrm{CH}_{3}-27\right), 2,27$ (1H, t-like, $\left.J=8,4 \mathrm{~Hz}, \mathrm{H}-17\right), 2,37$ $(1 \mathrm{H}, \mathrm{dd}, J=3,9,12,1 \mathrm{~Hz}, \mathrm{H}-5), 3,05$ (1H, m, H-9), 3,33 (1H, m, H-22), 3,97 (1H, br s, H-3), 5,78 (1H, s, H-7). ESMS (+ve): m/z (\% rel. intensity) 465,2 [M+H] ${ }^{+}(100)$.

На ВЭЖХ этот компонент экдистероидной суммы имеет время удерживания 15,303 мин, максимум поглощения $\lambda=248$ нм, в масс-спектре характерную фрагментацию $\left(\mathrm{M}^{+} 464\right)$ и идентифицирован с 2дезокси-20-гидроксиэкдизоном.

20,26-дигидроксиэкдизон (V). Соединение со временем удерживания 8,652 мин, имеющее свойственный экдистероидам УФ-спектр ( $\lambda=248$ нм) и масс-спектр с характерной фрагментацией $\left(\mathrm{M}^{+} 496\right)$, соответствует структуре 20,26-дигидроксиэкдизона. Это подтверждается спектром ПМР $\left(\mathrm{CD}_{3} \mathrm{OD}, \delta\right.$, м.д.): 0,930 (3H, c, $\left.\mathrm{CH}_{3}-18\right), 1,109$ (3H, c, $\left.\mathrm{CH}_{3}-19\right), 1,222$ (3H, c, $\left.\mathrm{CH}_{3}-21\right), 1,222$ (3H, c, $\left.\mathrm{CH}_{3}-27\right), 5,877$ (1H, д., H-7), 2,607 (1Н, дд., Н-5), 3,114 (1Н, т., Н-9), 2,415 (1Н, т., Н-17), 3,345 ( $\mathrm{CH}_{2}-\mathrm{OH}$, дд).

Компонент экдистероидной суммы со временем удерживания 9,298 мин, имеющий характерный для экдистероидов УФ-спектр $\left(\lambda=248\right.$ нм) и масс-спектр, свойственный для экдистероида с $\mathrm{M}^{+} 512$, соответствует структуре 26-гидроксиполиподина В (VI).

Соединение со временем удерживания 16,401 мин, имеющий в УФ-спектре $\lambda=248$ нм и масс-спектр с характерной фрагментацией $\left(\mathrm{M}^{+} 416\right)$, что соответствует структуре 2-дезоксиэкдизона (VII).

20-гидроксиэкдизон-25- $\beta$-D-гликозид (VIII), имеет УФ-спектр ( $\lambda=248$ нм) и масс-спектр с характерной фрагментацией $\left(\mathrm{M}^{+}\right.$643). Спектр ПМР (ДМСО, $\delta$, м.д.): 0,752 (3H, c, $\left.\mathrm{CH}_{3}-18\right), 0,851$ (3H, c, $\left.\mathrm{CH}_{3}-19\right)$, 1,069 (3H, c, $\left.\mathrm{CH}_{3}-21\right), 1,045$ (3H, c, $\left.\mathrm{CH}_{3}-26\right), 1,054$ (3H, c, $\left.\mathrm{CH}_{3}-27\right), 1,468$ (1H, д., Н-1), 5,642 (1H, д., Н-7), 2,150 (1Н, дд., Н-5), 1,494 (2Н, т., Н-4), 2,980 (1Н, т., Н-9), 1,707 (2Н, т., Н-15), 1,633 (2Н, дд., Н-16), 2,244 (1H, т., H-17), 4,636 (1H, д., glu - H1), 3,115 (1H, glu - H2), 3,523 (1H, т., glu - H3), 3,485 (1H, glu - H4), 4,413 (2H, дд., glu - H6). По данным хроматографического (ВЭЖХ/МС) и спектрального (ЯМР) анализа это соединение можно идентифицировать как 20-гидроксиэкдизон-25- $\beta$-D-гликозид [5]. Данное соединение из корней Silene frivaldszkyana было выделено впервые. Ранее экдистероид VIII был обнаружен в Pfaffia iresinoides (Amaranthaceae) [7] и Silene otites (Caryophyllaceae) [8].

26-гидроксиинтегристерон А (IX) в бутанольном экстракте корней Silene frivaldszkyana идентифицирован ВЭЖХ на основании времени удерживания искомого соединения, выделенного из надземной части [3].

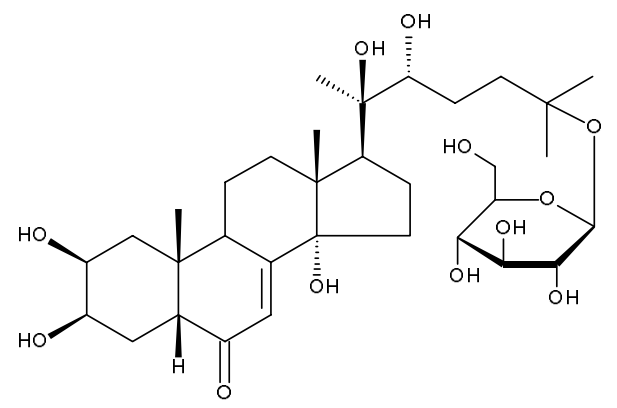

VIII

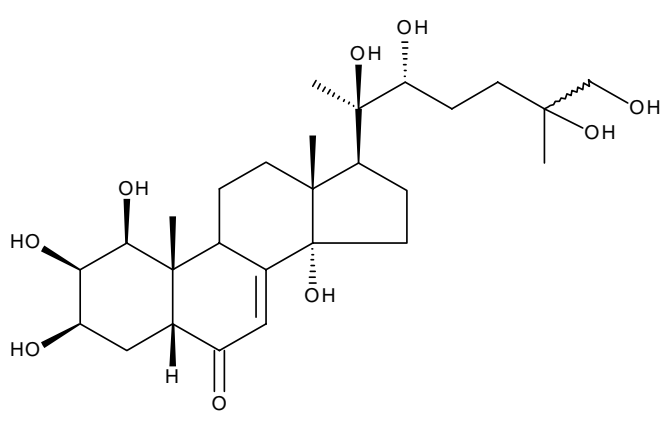

IX

Структуры некоторых экдистероидов, выделенных из корней Silene frivaldszkyana:

VIII - 20-гидроксиэкдизон-25- $\beta$-D-глюкозид; IX - 26-гидроксиинтегристерон А 
Сравнение состава выделенных экдистероидов из надземной и подземной частей Silene frivaldszkyana показало, что корни как запасающий орган аккумулируют дополнительно глюкозид 20гидроксиэкдизона, по всей вероятности, являющийся запасной формой экдистероидов зимой в корнях многолетних растений и предшественником 20-гидроксиэкдизона и всех других экдистероидов в процессе начала биосинтеза весной в листьях и других органах надземной части Silene frivaldszkyana.

\section{Выводы}

1. В корнях Silene frivaldszkyana, интродуцированной в Западной Сибири, обнаружено методом ВЭЖХ/МС больше экдистероидов, чем в надземной части.

2. Выделено и идентифицировано из корней Silene frivaldszkyana 9 экдистероидов: 20-гидроксиэкдизон, полиподин В, 2-дезокси-20-гидроксиэкдизон, 2-дезоксиэкдизон, интегристерон А, 26-гидроксиполиподин В, 20,26-дигидроксиэкдизон, 20-гидроксиэкдизон-25- $\beta$-D-гликозид, 26-гидроксиинтегристерон А.

\section{Список литературы}

1. Зибарева Л.Н., Еремина В.И., Иванова Н.А. Новые экдистероидоносные виды рода Silene L. и динамика содержания в них экдистерона // Растительные ресурсы. 1997. Т. 33, вып. 3. С. 73-76.

2. Zibareva L. Distribution and levels of phytoecdysteroids in plants of genus Silene during development // Archives of insect biochemistry and physiology. 2000. Vol. 43. Pp. 1-8.

3. Zibareva L., Yeriomina V. I., Munkhjargal N., Girault J.-P., Dinan L.,. Lafont R. The Phytoecdysteroid Profiles of 7 Species of Silene (Caryophyllaceae) // Archives of insect biochemistry and physiology. 2009. Vol. 72. N4. Pp. 234-248.

4. Патент № 2445110 (РФ). Способ получения 26-интегристерона А из растительного сырья / Л.Н. Зибарева, О.В. Волкова / 07.12.2010.

5. Ecdybase [Electronic resource]. URL: http://www.ecdybase.org

6. Зибарева Л.Н., Волкова О.В., Лафон Р. Виды рода Silene - продуценты 26-гидроксиэкдистероидов // Теоретическая и прикладная экология. 2012. №1. С. 66-72.

7. Nishimoto N., Shiobara Y., Inoue S., Fujino M. Three ecdysteroid glycosides from Pfaffia iresinoides // Phytochemistry. 1988. Vol. 27. Pp. 1665-1668.

8. Girault J.-P., Bathori M., Varga E., Szendrei K., Lafont R. Isolation and identification of new ecdysteroids from the Caryophyllaceae // J. of Natural Products. 1990. Vol. 53. Pp. 279-293.

Поступило в редакиฺию 19 июля 2016 г. 
Zibareva L.N. ${ }^{1}$, Volkova O.V. ${ }^{l}$, Morozov S.V. ${ }^{2}$, Chernayk E.I. ${ }^{2}$ PHYTOECDYSTEROIDS OF ROOT SILENE FRIVALDSZKYANA HAMPE

${ }^{1}$ Tomsk State University, Siberian Botanical Garden, pr. Lenina, 36, Tomsk, 634050 (Russia),

e-mail: zibareva.lara@yandex.ru

${ }^{2}$ Novosibirskij Institute of organic chemistry of SB RAS, pr.Lavrenteva 9, Novosibirsk, 630090 (Russia)

Ecdysteroids were isolated from roots of Silene frivaldszkyana (Caryophyllaceae) introduced into culture in Western Siberia: 20-hydroxyecdysone, polypodine B, integristerone A, 2-deoxy-20-hydroxyecdysone, 2-deoxyecdysone, 20,26dihydroxyecdysone, 26-hydroxypolypodine B, 26-hydroxyintegristerone A, 20-hydroxyecdysone-25- $\beta$-D-glucoside. Structures are installed using NMR, mass spectroscopy, HPLC. The presence of 26-hydroxyintegristerone A in the roots established by comparative HPLC analysis with compound isolated from overground part of plants.

It is shown that this Silene species synthesizes characteristic for this genus of ecdysteroids: 2-deoxy-20hydroxyecdysone, 2-deoxyecdysone, 20-hydroxyecdysone, polypodine B, integristerone A, and is a rich source of their 26hydroxy derivatives. Among the common ecdysteroids of the genus Silene 20-hydroxyecdysone-25- $\beta$-D-glycoside was isolated for the first from roots of $S$. frivaldszkyana.

Comparative analysis of ecdysteroids in aboveground and underground parts showed that the roots contain a richer composition of them that is likely so that the roots of perennial plants accumulate these compounds. And 20-hydroxyecdysone$25-\beta$-D-glycoside is a spare substance of major component 20-hydroxyecdysone.

Keywords: Phytoecdysteroids, identification, roots of Silene frivaldszkyana

\section{References}

1. Zibareva L.N., Eremina V.I., Ivanova N.A. Rastitel'nye resursy, 1997, vol. 33, no. 3, pp. 73-76. (in Russ.).

2. Zibareva L. Archives of insect biochemistry and physiology, 2000, vol. 43, pp. 1-8.

3. Zibareva L., Yeriomina V. I., Munkhjargal N., Girault J.-P., Dinan L.,. Lafont R. Archives of insect biochemistry and physiology, 2009, vol. 72, no. 4, pp. 234-248.

4. Patent 2445110 (RU). 07.12.2010. (in Russ.).

5. Ecdybase [Electronic resource]. URL: http://www.ecdybase.org

6. Zibareva L.N., Volkova O.V., Lafon R. Teoreticheskaia i prikladnaia ekologiia, 2012, no. 1, pp. 66-72. (in Russ.).

7. Nishimoto N., Shiobara Y., Inoue S., Fujino M. Phytochemistry, 1988, vol. 27, pp. 1665-1668.

8. Girault J.-P., Bathori M., Varga E., Szendrei K., Lafont R. J. of Natural Products, 1990, vol. 53, pp. $279-293$.

Received July 19, 2016

\footnotetext{
* Corresponding author.
} 
